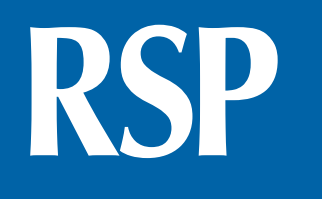

http://www.rsp.fsp.usp.br/
Revista de Saúde Pública

\title{
Desempenho e percepção sobre modelos de rotulagem nutricional frontal no Brasil
}

\author{
Luisete Moraes Bandeira' iD, Jéssica Pedroso" (iD, Natacha Toral" (iD, Muriel Bauermann Gubert'," \\ ' Universidade de Brasília. Faculdade de Ciências da Saúde. Programa de Pós-Graduação em Saúde Coletiva. \\ Asa Norte, Campus Universitário Darcy Ribeiro, Brasília, DF, Brasil \\ " Faculdade de Ciências da Saúde. Programa de Pós-Graduação em Nutrição Humana. Brasília, DF, Brasil
}

\section{RESUMO}

OBJETIVO: Avaliar o desempenho e a percepção de cinco modelos de rotulagem nutricional frontal (RNF) entre consumidores brasileiros.

MÉTODOS: Estudo transversal, com aplicação de questionário online a 2.400 indivíduos, alocados de forma aleatória em seis grupos de estudo, sendo um controle e cinco expostos a modelos de RNF (octógono, triângulo, círculo, lupa e semáforo), aplicados a nove alimentos. Foi avaliado o entendimento do conteúdo nutricional, a percepção de saudabilidade, a intenção de compra e a percepção dos consumidores brasileiros sobre os modelos.

RESULTADOS: Todos os modelos de RNF aumentaram o entendimento do conteúdo nutricional e reduziram a percepção de saudabilidade e a intenção de compra, quando comparados ao grupo controle (41,3\%). Os modelos de RNF de advertência - octógono (62,4\%), triângulo (61,9\%) e círculo $(61,8 \%)$ - apresentaram desempenho significativamente superior ao semáforo $(55,0 \%)$ quanto ao entendimento do conteúdo nutricional. O desempenho do modelo da lupa $(59,5 \%)$ não diferiu dos outros quatro modelos testados, inclusive do semáforo (55,0\%), para o entendimento do conteúdo nutricional. A análise individual dos alimentos sugere melhor desempenho das advertências em relação à lupa e ao semáforo para a percepção de saudabilidade e a intenção de compra. Os consumidores manifestam-se favoráveis à presença da RNF, percebendo-a como confiável para aumentar o entendimento das informações nutricionais.

Universidade de Brasília

Faculdade de Ciências da Saúde Campus Universitário Darcy Ribeiro 70910-900 Brasília, DF, Brasil

E-mail: luisetebandeira@gmail.com

Recebido: 15 fev 2020

Aprovado: 16 jul 2020

Como citar: Bandeira LM, Pedroso J, Toral N, Gubert MB.

Desempenho e percepção sobre modelos de rotulagem nutricional frontal no Brasil. Rev Saude

Publica. 2021;55:19.

https://doi.org/10.11606/s1518-

8787.2021055002395

Copyright: Este é um artigo de acesso aberto distribuído sob os termos da Licença de Atribuição Creative Commons, que permite uso irrestrito, distribuição e reprodução em qualquer meio, desde que o autor e a fonte originais sejam creditados.
CONCLUSÃO: A RNF deve ser implementada nos rótulos dos alimentos no Brasil, considerando que aumenta o entendimento nutricional, reduz a percepção de saudabilidade e a intenção de compra de alimentos com nutrientes críticos. As advertências apresentaram melhor desempenho quando comparadas aos demais modelos.

DESCRITORES: Rotulagem Nutricional Frontal. Rotulagem Nutricional. Rotulagem de Alimentos. Aviso. Política Nutricional, Promoção da Saúde. 


\section{INTRODUÇÃO}

A rotulagem nutricional frontal (RNF) é recomendada internacionalmente ${ }^{1}$ como ferramenta para auxiliar o consumidor na interpretação das declarações quantitativas de nutrientes dos alimentos, geralmente de difícil entendimento e dispostas em letras pequenas no verso das embalagens ${ }^{2}$. Quase metade da população brasileira têm dificuldade de interpretar a informação nutricional dos rótulos dos alimentos ${ }^{3}$. Ao não compreender o conteúdo do alimento, o julgamento quanto à saudabilidade e, consequente, a decisão de compra do indivíduo são afetados ${ }^{4,5}$.

Vários países adotam diferentes modelos de RNF para ajudar o consumidor nessa interpretação. Os modelos de advertência (octógono, círculo e triângulo), informam, de maneira simples e direta, se o alimento apresenta alto teor de algum nutriente (açúcares, gorduras, sódio). Eles têm se mostrado mais eficientes em aumentar o entendimento, e consequentemente, reduzir a percepção de saudabilidade e a intenção de compra dos alimentos, quando comparados ao semáforo nutricional, que informa o baixo, médio e alto conteúdo dos nutrientes, ou ao Guideline Daily Amounts (GDA), que indica o percentual de nutriente presente no alimento em relação ao valor diário recomendado ${ }^{6-10}$. Nos últimos anos, quatro países da América Latina - Chile, Peru, Uruguai e México - adotaram a RNF de advertência no formato de octógono como obrigatória ${ }^{11-14}$.

No Brasil, a Agência Nacional de Vigilância Sanitária (Anvisa) aprovou, em 2020, um modelo de RNF em formato retangular preto com lupa, semelhante ao que vem sendo discutido no Canadá15,16. Contudo, apenas dois estudos avaliaram o desempenho deste modelo de RNF, sendo ele inferior ao octógono e ao triângulo em reduzir o tempo para identificação de nutrientes presentes em excesso, entre adultos brasileiros 9 . O modelo da lupa também se mostrou inferior ao octógono, círculo e triângulo em aumentar o entendimento do conteúdo nutricional entre adultos dos Estados Unidos, Canadá, Austrália e Reino Unido ${ }^{17}$.

O desempenho dos modelos de RNF pode, ainda, ser influenciado por fatores como a motivação para a saúde, a facilidade de preparo e o preço ${ }^{18}$, bem como por aspectos relacionados ao próprio design do modelo, como sua capacidade e chamar atenção, a facilidade do consumidor em identificar e processar sua informação, a familiaridade com a RNF e a percepção de risco por ela gerada ${ }^{8,19-21}$.

Em virtude disso, é importante a realização de estudos locais para identificar o modelo de RNF mais adequado para a população de cada país ${ }^{22}$. Observa-se a necessidade de estudos que comparem o desempenho de diferentes modelos de RNF no Brasil, incluindo a lupa, investigada em apenas um dos dois estudos realizados com adultos brasileiros que compararam mais de um modelo de $\mathrm{RNF}^{9,10}$.

Diante disso, o objetivo deste estudo foi avaliar o desempenho de cinco modelos de rotulagem nutricional frontal (octógono, triângulo, círculo, lupa e semáforo) em aumentar o entendimento do conteúdo nutricional, reduzir a percepção de saudabilidade e a intenção de compra de alimentos, além de identificar a percepção de consumidores adultos brasileiros sobre estes modelos e a importância de fatores relacionados à escolha alimentar.

\section{MÉTODOS}

\section{Participantes do Estudo}

Foi realizado estudo transversal com uma amostra de 2.400 indivíduos alocados de forma aleatória em seis grupos de estudo. A amostra foi feita por cotas, sendo representativa da população brasileira em relação a sexo, classe econômica e às cinco macrorregiões do país. O recrutamento dos participantes foi realizado de forma digital, por uma empresa especializada em pesquisas online que possui cadastro dos respondentes. Os convites 
foram enviados apenas para pessoas que atendiam ao perfil de cotas pré-determinado na amostra. À medida que as cotas eram finalizadas, os convites eram enviados para as demais cotas.

O questionário foi aplicado em agosto de 2019. Todos os indivíduos assentiram em sua participação por meio do Termo de Consentimento Livre e Esclarecido. O estudo foi aprovado pelo Comitê de Ética em Pesquisa com Seres Humanos da Faculdade de Ciências da Saúde da Universidade de Brasília (protocolo 67420817.7.0000.0030).

\section{Alocação da Amostra nos Grupos de Pesquisa}

Os participantes foram alocados de forma aleatória em seis grupos, sendo 400 no grupo controle (CG) e os demais em um dos cinco grupos de exposição: 1) lupa (n=400); 2) círculo $(\mathrm{n}=400)$; 3) octógono $(\mathrm{n}=400)$, 4) triângulo $(\mathrm{n}=400)$ e 5) e semáforo nutricional $(\mathrm{n}=400)$ (Figura 1). A inclusão de um grupo controle permitiu a comparação do desempenho dos modelos de RNF entre si e o desempenho de cada um individualmente em relação à ausência de RNF no alimento.

\section{Posição e Tamanho dos Modelos de RNF}

Os modelos de RNF (Figura 1) foram aplicados no canto superior direito, em diferentes percentuais da área do painel principal do alimento: $15 \%$ se o alimento tinha alto conteúdo de açúcares, sódio e gordura saturada; $10 \%$ quando alto em dois destes nutrientes; e $5 \%$ em um destes nutrientes. Para a lupa foi sempre utilizada $10 \%$ da área.

A lupa, o octógono e o triângulo foram apresentados na cor preta e o círculo na cor vermelha. O semáforo nutricional foi apresentado nas cores vermelha, amarela e verde, apontando respectivamente os teores de alto, médio ou baixo dos nutrientes.

Para a definição do baixo, médio e alto teor de nutrientes (açúcares livres, gordura saturada e sódio) foi adotado o modelo de perfil nutricional mais restritivo proposto pela Anvisa ${ }^{23}$.

\section{Seleção dos Alimentos}

Um painel de especialistas selecionou nove alimentos comumente consumidos pela população brasileira ${ }^{24}$ e usualmente percebidos como saudáveis, apesar de apresentarem alto conteúdo de, pelo menos, um nutriente (açúcares livres, gordura e sódio) (Tabela 1).
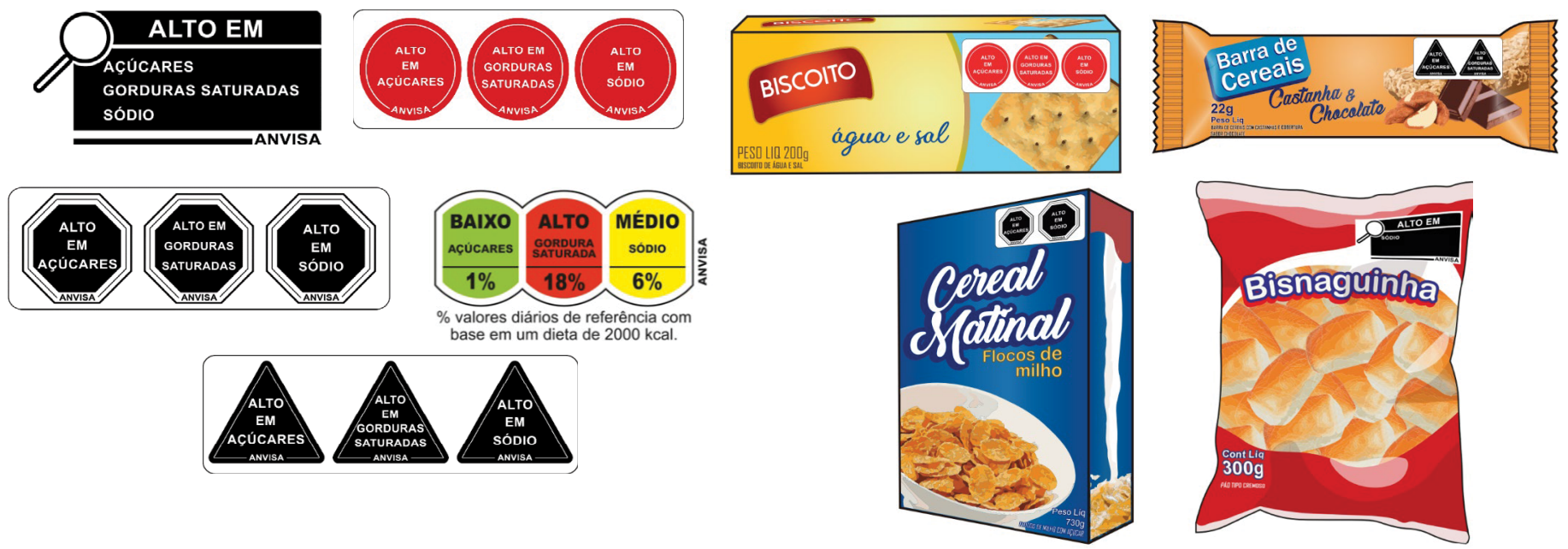

Notas: Lupa preta - modelo proposto pela Gerência Geral de Alimentos da Agência Nacional de Vigilância Sanitária; círculo vermelho - modelo proposto pela Fundação Ezequiel Dias; preto - modelo proposto pela Câmara Interministerial de Segurança Alimentar e Nutricional; triângulo preto - modelo proposto pelo instituto Brasileiro de Defesa do Consumidor e Universidade Federal do Paraná; semáforo nutricional - modelo proposto pelo setor de indústria de alimentos e bebidas por meio da Rede de Rotulagem.

Figura. Modelos de rotulagem nutricional frontal testados e exemplos das imagens dos alimentos visualizadas pelos participantes. Brasil, 2019. 
Tabela 1. Composição nutricional dos produtos incluídos no estudo e teor (baixo, médio e alto) dos nutrientes associados às doenças crônicas não transmissíveis, de acordo com os critérios estabelecidos no relatório preliminar de análise de impacto regulatório sobre rotulagem nutricional. Gerência Geral de Alimentos. Agência Nacional de Vigilância Sanitária, 2018.

\begin{tabular}{|c|c|c|c|c|c|c|c|c|}
\hline \multirow[b]{2}{*}{ Alimento } & \multirow{2}{*}{$\begin{array}{c}\text { Tamanho da } \\
\text { porção } \\
(\mathrm{g} / \mathrm{mL})\end{array}$} & \multicolumn{4}{|c|}{ Conteúdo de nutrientes por porção } & \multicolumn{3}{|c|}{ Classificação do conteúdo de nutriente } \\
\hline & & $\begin{array}{c}\text { Calorias } \\
\text { (Kcal) }\end{array}$ & $\begin{array}{l}\text { Açúcares } \\
\text { livres (g) }\end{array}$ & $\begin{array}{c}\text { Gorduras } \\
\text { saturadas (g) }\end{array}$ & Sódio (mg) & $\begin{array}{l}\text { Açúcares } \\
\text { livres }\end{array}$ & $\begin{array}{l}\text { Gorduras } \\
\text { saturadas }\end{array}$ & Sódio \\
\hline Requeijão & 30 & 81 & 7 & 4,1 & 144 & Médio & Alto & Alto \\
\hline Molho de tomate & 60 & 30 & 5,8 & 0 & 311 & Baixo & Baixo & Alto \\
\hline Pão de forma & 50 & 133 & 20 & 0,5 & 219 & Baixo & Baixo & Alto \\
\hline Biscoito água e sal & 30 & 129 & 20 & 1,7 & 210 & Alto & Alto & Alto \\
\hline Barra de cereal & 22 & 91 & 16 & 1,6 & 17 & Alto & Alto & Baixo \\
\hline Cereal matinal & 30 & 111 & 25 & 0 & 147 & Alto & Baixo & Alto \\
\hline Néctar & 200 & 40 & 10 & 0 & 0 & Alto & Baixo & Baixo \\
\hline Milho verde & 130 & 129 & 25 & 0 & 606 & Baixo & Baixo & Alto \\
\hline Bisnaguinha & 40 & 124 & 22 & 0,8 & 189 & Médio & Médio & Alto \\
\hline
\end{tabular}

Conforme realizado em estudo anterior ${ }^{9}$, as imagens dos alimentos foram elaboradas por empresa especializada em design gráfico exclusivamente para esta pesquisa e não continham alegações de saúde, marcas ou nomes comerciais, buscando neutralizar a influência destes fatores no desempenho dos modelos (Figura 1).

\section{Coleta de Dados}

O questionário foi organizado em três seções: 1) características dos participantes, 2) desempenho dos modelos de RNF e 3) percepção dos consumidores em relação aos modelos de RNF.

Na seção 1, foram identificadas as características dos participantes (sexo, faixa etária, escolaridade, renda e região de moradia), incluindo a importância de fatores relacionados à escolha alimentar. Foram elaborados dez itens baseados no Food Choice Questionnaire ${ }^{18}$, para os quais os participantes avaliaram a importância com opções de respostas em escala Likert de 5 pontos, variando de: 1 - "nem um pouco importante" a 5 - "muito importante". Os itens eram "Eu escolho alimentos": a) mais fáceis de preparar; b) que o local de compra é próximo para mim; c) pelo preço; d) que são mais saudáveis; e) que me deixam alegre, relaxado, ativo/acordado; f) naturais, sem aditivos ou ingredientes artificiais/industrializados; g) com poucas calorias, açúcares ou gorduras; h) da marca que sempre costumo comprar; i) ou comidas parecidas com o que comia na infância; j) que não prejudiquem o meio ambiente, dando preferência aos alimentos orgânicos e evitando alimentos com agrotóxicos.

Na seção 2, foi avaliado o desempenho dos modelos de RNF em aumentar o entendimento do conteúdo nutricional e em reduzir a percepção de saudabilidade e a intenção de compra dos alimentos apresentados. Os indivíduos visualizavam, individualmente e em ordem aleatória, os nove alimentos com o modelo de RNF segundo seu grupo de randomização. Nenhum indivíduo foi exposto a mais de um tipo de RNF. Enquanto visualizavam cada alimento, os participantes responderam a três perguntas. A primeira pergunta mensurava o entendimento do conteúdo nutricional do alimento: "na sua opinião, esse alimento contém nutrientes em niveis mais altos do que o recomendado para uma dieta saudável?”. Para fins de padronização e comparação entre os modelos de RNF estudados, optou-se por manter apenas essa pergunta para o semáforo, uma vez que esse era o único modelo de RNF que permitia quantificar níveis médio e baixo de nutrientes ${ }^{9,10}$. As opções de resposta eram de múltipla escolha: "muito açúcar", "muito sódio", "muita gordura saturada" ou "não contém nenhum nutriente em muita quantidade", sendo possível que o participante marcasse mais de uma opção de reposta. Outras duas perguntas mensuraram a intenção de compra e a percepção de saudabilidade dos produtos, com opções de resposta em escala Likert de 5 pontos: "você compraria este alimento?" (1 - "eu certamente não compraria" 
a 5 - "eu certamente compraria"); e "você considera este alimento": (1 - "nada saudável" a 5 - "muito saudável”). O grupo controle visualizou os mesmos alimentos, porém, sem qualquer modelo de RNF. Caso desejassem, os sujeitos de todos os grupos podiam visualizar a tabela de informação nutricional e a lista de ingredientes de cada alimento ao clicar em um botão disposto logo abaixo da imagem do alimento.

Na seção 3, foi avaliada a percepção dos participantes em relação aos modelos de RNF em relação à "facilidade de identificação", à "confiabilidade", ao "processamento da informação" e à "preferência”. Essas dimensões foram baseadas na estrutura de aceitabilidade proposta por Nielsen ${ }^{25}$, já utilizada em estudos sobre $\mathrm{RNF}^{19,20}$. As perguntas eram apresentadas enquanto o participante visualizava o modelo de RNF do seu grupo de randomização, de forma isolada. Para avaliar a facilidade de identificação, o indivíduo respondeu às seguintes perguntas/afirmação: "1. Você viu que havia esse selo no alimento que você avaliou? (sim ou não); “2. foi difícil visualizar esse selo no alimento" "3. encontrei mais rapidamente as informações nutricionais com esse selo". Para "confiabilidade", a afirmativa era "4. confiei nas informações deste selo". Quando avaliado o processamento da informação, as seguintes afirmações foram apresentadas - "5. entendi mais rapidamente as informações nutricionais com esse selo", - "6. entendi esse selo", "7. me senti desconfortável com esse selo". A seguinte afirmação permitiu avaliar a preferência: "8. eu gostaria de encontrar esse selo nas embalagens de alimentos".

Ainda nessa seção foi investigado se os modelos de RNF induziram os participantes à emoção básica medo ${ }^{25}$ por meio da afirmação: "9. a presença desse selo me causou medo". As questões 2 à 9 tinham opções de resposta em escala do tipo Likert de 5 pontos, variando de 1 - "discordo totalmente" a 5 - "concordo totalmente". Esta seção foi apresentada apenas para os participantes dos grupos exposição.

\section{Análises Estatísticas}

Para o cálculo do tamanho amostral foi considerado nível de confiança de 95\%, erro máximo aceitável de 2 pontos percentuais, alfa de 0,05 e poder do teste de $95 \%$. No cálculo da amostra foi considerada a média de acertos para entendimento do conteúdo nutricional de $57,6 \%$ para o semáforo e de 79,9\% para o triângulo ${ }^{10}$ e a utilização do teste ANOVA one-way, estimando-se um tamanho de efeito de 11. Assim, este estudo deveria incluir pelo menos 210 adultos por grupo, aos quais foram adicionados $100 \%$ para cobertura de possível perda ou inconsistência de dados, estimando-se uma amostra de 2.400 indivíduos. O cálculo foi realizado pelo programa $G^{*}$ Power 3.1.9.2.

Para o entendimento da RNF, primeiramente foi calculado o percentual de acertos de itens para cada alimento de acordo com a resposta do participante em relação à presença ou à ausência do nutriente em excesso. Para o conjunto dos nove alimentos, considerou-se o percentual de acertos do participante em relação a todos os alimentos. Posteriormente, foram comparadas as médias do percentual de acertos dos grupos de exposição e grupo controle.

Foram calculadas também, para os seis grupos, as médias de intenção de compra e de percepção de saudabilidade dos participantes em relação aos nove alimentos em conjunto e individualmente. A visualização do modelo de RNF foi expressa em percentual. Também foram calculadas as médias de concordância, segundo a escala do tipo Likert, considerada como variável contínua, para as questões que avaliaram a percepção dos participantes em relação aos modelos de RNF.

Utilizou-se teste qui-quadrado de Pearson (variáveis categóricas) ou ANOVA one-way com pós teste Tukey (variáveis contínuas) para verificar se existiam diferenças entre os grupos quanto às características dos participantes, o desempenho dos modelos de RNF e a percepção dos participantes em relação aos modelos de RNF entre os grupos. Foi considerado o intervalo de confiança de $95 \%$. Todas as análises foram conduzidas por meio do software Statistical Package for the Social Sciences (SPSS), versão 23.0. 


\section{RESULTADOS}

A maioria da amostra de 2.400 adultos tinha idade entre 18 e 34 anos (55,1\%), 51,2\% eram mulheres e $37,1 \%$ possuíam ensino médio completo. As características dos participantes não apresentaram diferença estatística entre os seis grupos de pesquisa.

A média de importância dos fatores relacionados à escolha alimentar (facilidade de preparo, proximidade do local de compra, preço, preferência por alimentos mais saudáveis, com conteúdo natural, para controle de peso e a preocupação ética na escolha do alimento) atribuída pelos participantes não diferiu entre os grupos controle e exposição (Tabela 2).

Tabela 2. Características sociodemográficas dos participantes do estudo e média de importância atribuída aos fatores relacionados à escolha alimentar. Brasil, 2019.

\begin{tabular}{|c|c|c|c|c|c|c|c|c|}
\hline Características & $\begin{array}{c}\text { Amostra } \\
\text { total } \\
\mathrm{n}=2.400 \\
(\%)\end{array}$ & $\begin{array}{c}\text { Lupa } \\
\mathrm{n}=400 \\
(\%)\end{array}$ & $\begin{array}{c}\text { Círculo } \\
\mathrm{n}=400 \\
(\%)\end{array}$ & $\begin{array}{c}\text { Octógono } \\
\mathrm{n}=\mathbf{4 0 0} \\
(\%)\end{array}$ & $\begin{array}{c}\text { Semáforo } \\
\mathrm{n}=400 \\
(\%)\end{array}$ & $\begin{array}{c}\text { Triângulo } \\
n=400 \\
(\%)\end{array}$ & $\begin{array}{c}\text { Controle } \\
\mathrm{n}=400 \\
(\%)\end{array}$ & $\mathbf{p}$ \\
\hline
\end{tabular}

Sexo

$\begin{array}{lcccccccc}\begin{array}{l}\text { Masculino } \\ \text { Feminino }\end{array} & 48,8 & 48,8 & 48,0 & 47,8 & 49,5 & 49,5 & 49,3 & 0,993^{\text {a }} \\ \text { Faixa etária, } & 51,2 & 51,3 & 52,0 & 52,3 & 50,5 & 50,5 & 50,8 & \\ 18-34 & & & & & & & & \\ 35-54 & 55,1 & 52,8 & 54,8 & 56,5 & 55,5 & 53,0 & 58,0 & \\ \geq 55 & 35,9 & 37,8 & 35,5 & 35,5 & 35,5 & 37,0 & 34,0 & 0,940^{\text {a }} \\ & 9,0 & 9,5 & 9,8 & 8,0 & 9,0 & 10,0 & 8,0\end{array}$

Escolaridade, em anos

$\begin{array}{lcccccccr}<9 & 3,3 & 3,3 & 4,8 & 3,5 & 2,0 & 3,8 & 2,3 & \\ 9<12 & 10,8 & 9,5 & 12,0 & 9,8 & 11,8 & 10,0 & 11,8 & 0,510^{\text {a }} \\ \geq 12 & 86,0 & 87,3 & 83,3 & 86,8 & 86,3 & 86,3 & 86,0 & \end{array}$

Renda, em salários-mínimos ${ }^{b}$

$\begin{array}{lccccccccc}<2 & 48,4 & 45,8 & 47,5 & 48,8 & 49,0 & 49,8 & 49,5 & \\ 2<10 & 46,1 & 48,3 & 46,0 & 48,3 & 45,3 & 43,8 & 45,3 & 0,569^{\text {a }} \\ \geq 10 & 5,5 & 6,0 & 6,5 & 3,0 & 5,8 & 6,5 & 5,3 & \end{array}$

Região

$\begin{array}{lcccccccc}\text { Norte } & 5,1 & 4,5 & 5,25 & 5,5 & 4,75 & 5,25 & 5,75 & 7,75 \\ \text { Centro-Oeste } & 8,3 & 8,5 & 7,5 & 9 & 8,5 & 9 & 22,5 & 1,000^{\text {a }} \\ \text { Nordeste } & 23 & 24,5 & 23 & 21,8 & 22,3 & 24,5 & 22,5 \\ \text { Sudeste } & 47,8 & 46,5 & 48 & 48,3 & 50,2 & 45,5 & 48,5 & 15,75\end{array}$

Importância de fatores relacionados à escolha alimentar - "eu escolho alimentos:"

\begin{tabular}{|c|c|c|c|c|c|c|c|}
\hline Mais fáceis de prepararc & 3,69 & 3,69 & 3,51 & 3,67 & 3,67 & 3,80 & $0,049^{d}$ \\
\hline Que o local de compra é próximo para mimc & 3,86 & 3,66 & 3,83 & 3,80 & 3,83 & 3,77 & $0,226^{d}$ \\
\hline Pelo preço ${ }^{c}$ & 3,72 & 3,70 & 3,84 & 3,74 & 3,77 & 3,86 & $0,250^{d}$ \\
\hline Que são mais saudáveis ${ }^{c}$ & 4,15 & 4,11 & 4,11 & 4,09 & 4,16 & 4,09 & $0,874^{\mathrm{d}}$ \\
\hline Que me deixam alegre, relaxado, ativo/acordado ${ }^{c}$ & 3,65 & 3,57 & 3,64 & 3,65 & 3,65 & 3,77 & $0,320^{\mathrm{d}}$ \\
\hline Naturais, sem aditivos ou ingredientes artificiais/industrializados ${ }^{c}$ & 3,68 & 3,64 & 3,72 & 3,62 & 3,67 & 3,64 & $0,885^{\mathrm{d}}$ \\
\hline Com poucas calorias, açúcares ou gorduras ${ }^{c}$ & 3,63 & 3,66 & 3,65 & 3,59 & 3,63 & 3,63 & $0,978^{d}$ \\
\hline Da marca que sempre costumo comprar ${ }^{c}$ & 3,73 & 3,76 & 3,72 & 3,68 & 3,64 & 3,77 & $0,580^{d}$ \\
\hline Ou comidas parecidas com o que comia na infância ${ }^{c}$ & 2,90 & 3,01 & 3,08 & 2,99 & 2,99 & 3,07 & $0,410^{\mathrm{d}}$ \\
\hline $\begin{array}{l}\text { Que não prejudiquem o meio ambiente, dando preferência aos } \\
\text { alimentos orgânicos e evitando alimentos com agrotóxicos }{ }^{c}\end{array}$ & 3,59 & 3,75 & 3,69 & 3,66 & 3,61 & 3,61 & $0,397^{\mathrm{d}}$ \\
\hline
\end{tabular}

${ }^{a}$ p-valores do teste qui-quadrado de Pearson.

b Valor do saláriomínimo: R\$998,00 (novecentos e noventa e oito reais)/\$229,42 (duzentos e vinte e nove dólares e quarenta e dois centavos).

"Média de importância de fatores relacionados à escolha alimentar: 1 - "nem um pouco importante a 5 - muito importante".

${ }^{\mathrm{d}} \mathrm{p}$-valores da ANOVA. 


\section{Entendimento do Conteúdo Nutricional}

Em relação ao percentual médio de acertos dos participantes para o conjunto de nove alimentos, todos os modelos de RNF tiveram desempenho significativamente superior ao GC (Tabela 3). Na presença do octógono, círculo e triângulo, os percentuais de acerto foram significativamente superiores ao percentual observado na presença do semáforo. O percentual médio de acertos na presença da lupa não foi diferente do percentual observado

Tabela 3. Desempenho de cinco modelos de rotulagem nutricional frontal em relação ao entendimento do conteúdo nutricional, percepção de saudabilidade e intenção de compra. Brasil, 2019.

\begin{tabular}{|c|c|c|c|c|c|c|c|}
\hline & $\begin{array}{c}\text { Lupa } \\
\mathrm{n}=\mathbf{4 0 0}\end{array}$ & $\begin{array}{l}\text { Círculo } \\
\mathrm{n}=\mathbf{4 0 0}\end{array}$ & $\begin{array}{l}\text { Octógono } \\
n=400\end{array}$ & $\begin{array}{l}\text { Semáforo } \\
\mathrm{n}=\mathbf{4 0 0}\end{array}$ & $\begin{array}{c}\text { Triângulo } \\
\mathbf{n}=\mathbf{4 0 0}\end{array}$ & $\begin{array}{l}\text { Controle } \\
\mathrm{n}=\mathbf{4 0 0}\end{array}$ & p \\
\hline \multicolumn{8}{|l|}{ Entendimento do conteúdo nutricional } \\
\hline $\begin{array}{l}\text { Percentual médio de acertos para o conjunto de nove } \\
\text { produtos }^{\text {a }}\end{array}$ & $59,5 \%$ b,c & $61,8 \%^{c}$ & $62,4 \%{ }^{c}$ & $55,0 \%{ }^{\mathrm{b}}$ & $61,9 \%^{c}$ & $41,3 \%^{a}$ & $<0,001$ \\
\hline Requeijão $^{b}$ & $64,6 \%{ }^{\mathrm{b}}$ & $68,6 \%{ }^{\mathrm{b}}$ & $67,5 \%{ }^{\mathrm{b}}$ & $63,8 \%{ }^{\mathrm{b}}$ & $66,6 \%{ }^{\mathrm{b}}$ & $51,7 \%{ }^{a}$ & $<0,001$ \\
\hline Molho de tomate ${ }^{b}$ & $72,6 \%{ }^{\mathrm{b}}$ & $75,5 \%{ }^{\mathrm{b}}$ & $77,1 \%{ }^{\mathrm{b}}$ & $70,2 \%{ }^{\mathrm{b}}$ & $75,3 \%{ }^{\mathrm{b}}$ & $56,7 \%{ }^{a}$ & $<0,001$ \\
\hline Pão de formab & $61,0 \%{ }^{\mathrm{c}}$ & $61,0 \%{ }^{c}$ & $61,1 \%{ }^{c}$ & $52,1 \%{ }^{\mathrm{b}}$ & $60,0 \% \mathrm{o}^{\mathrm{b}, \mathrm{c}}$ & $33,5 \% \%^{a}$ & $<0,001$ \\
\hline Biscoito água e sal ${ }^{b}$ & $50,6 \%{ }^{\mathrm{b}}$ & $54,0 \%{ }^{\mathrm{c}}$ & $54,5 \%^{c}$ & $46,3 \%{ }^{b}$ & $52,7 \%$ b,c & $29,0 \%{ }^{a}$ & $<0,001$ \\
\hline Barra de cereal ${ }^{b}$ & $46,7 \%{ }^{\mathrm{b}}$ & $46,6 \%{ }^{\mathrm{b}}$ & $47,6 \%{ }^{\mathrm{b}}$ & $45,5 \%{ }^{\mathrm{b}}$ & $47,3 \%{ }^{b}$ & $29,1 \%{ }^{a}$ & $<0,001$ \\
\hline Cereal matinal ${ }^{\mathrm{b}}$ & $39,1 \%{ }^{\mathrm{b}}$ & $42,0 \%{ }^{b}$ & $43,7 \%{ }^{b}$ & $38,6 \%{ }^{\mathrm{b}}$ & $42,0 \%{ }^{\mathrm{b}}$ & $30,3 \%{ }^{a}$ & $<0,001$ \\
\hline Néctar ${ }^{\mathrm{b}}$ & $70,2 \%{ }^{\mathrm{b}}$ & $72,0 \%{ }^{\mathrm{b}}$ & $73,9 \%{ }^{\mathrm{b}}$ & $70,7 \%{ }^{\mathrm{b}}$ & $68,4 \%{ }^{\mathrm{b}}$ & $50,4 \% \mathrm{o}^{\mathrm{a}}$ & $<0,001$ \\
\hline Milho verde ${ }^{b}$ & $72,7 \%{ }^{\mathrm{b}}$ & $74,2 \%{ }^{\mathrm{b}}$ & $75,0 \%{ }^{\mathrm{b}}$ & $62,6 \%{ }^{a}$ & $75,1 \%{ }^{\mathrm{b}}$ & $55,9 \%{ }^{a}$ & $<0,001$ \\
\hline Bisnaguinhab $^{\mathrm{b}}$ & $58,8 \%^{\mathrm{c}}$ & $63,2 \%{ }^{c}$ & $62,9 \%{ }^{c}$ & $49,4 \%{ }^{b}$ & $62,8 \%{ }^{c}$ & $37,2 \%{ }^{a}$ & $<0,001$ \\
\hline \multicolumn{8}{|l|}{ Percepção de saudabilidade } \\
\hline $\begin{array}{l}\text { Média de percepção de saudabilidade para o conjunto de } \\
\text { nove produtos }\end{array}$ & $3,09^{b}$ & $2,91^{\mathrm{b}}$ & $2,90^{\mathrm{b}}$ & $3,09^{b}$ & $2,94^{\mathrm{b}}$ & $3,40^{\mathrm{a}}$ & 0,001 \\
\hline Requeijão $^{d}$ & $3,00^{a, b}$ & $2,73^{\mathrm{b}}$ & $2,71^{\mathrm{b}}$ & $2,85^{\mathrm{b}}$ & $2,84^{\mathrm{b}}$ & $3,26^{\mathrm{a}}$ & 0,001 \\
\hline Molho de tomate ${ }^{d}$ & $2,98^{a, b, c}$ & $2,84^{c}$ & $2,79^{c}$ & $3,17^{a, b}$ & $2,89^{b, c}$ & $3,25^{\mathrm{a}}$ & 0,001 \\
\hline Pão de formad & $3,49^{b}$ & $3,34^{\mathrm{b}}$ & $3,38^{\mathrm{b}}$ & $3,64^{a}$ & $3,33^{\mathrm{b}}$ & $3,89^{a}$ & 0,001 \\
\hline Biscoito água e sal ${ }^{d}$ & $3,03^{b}$ & $2,78^{\mathrm{b}}$ & $2,85^{\mathrm{b}}$ & $2,89^{b}$ & $2,81^{\mathrm{b}}$ & $3,47^{\mathrm{a}}$ & 0,002 \\
\hline Barra de cereal ${ }^{d}$ & $3,24^{\mathrm{b}}$ & $3,05^{b}$ & $3,10^{b}$ & $3,09^{b}$ & $3,03^{b}$ & $3,66^{\mathrm{a}}$ & 0,001 \\
\hline Cereal matinal $^{\mathrm{d}}$ & $3,06^{a, b}$ & $2,81^{b}$ & $2,80^{\mathrm{b}}$ & $2,88^{\mathrm{b}}$ & $2,83^{b}$ & $3,34^{\mathrm{a}}$ & 0,001 \\
\hline Néctar ${ }^{d}$ & $3,03^{a, b}$ & $2,87^{\mathrm{b}}$ & $2,80^{b}$ & $3,11^{a, b}$ & $2,83^{b}$ & $3,30^{\mathrm{a}}$ & 0,001 \\
\hline Milho verde ${ }^{d}$ & $3,08^{a, b, c}$ & $2,94^{b, c}$ & $2,96^{b, c}$ & $3,35^{\mathrm{a}}$ & $3,04^{b, c}$ & $3,38^{\mathrm{a}}$ & 0,001 \\
\hline Bisnaguinha $^{d}$ & $2,94^{a, b}$ & $2,90^{a, b}$ & $2,76^{b}$ & $2,92^{a, b}$ & $2,91^{a, b}$ & $3,15^{a}$ & 0,001 \\
\hline \multicolumn{8}{|l|}{ Intenção de compra } \\
\hline $\begin{array}{l}\text { Média de intenção de compra para o conjunto de nove } \\
\text { produtos }^{\mathrm{e}}\end{array}$ & $3,39^{b}$ & $3,28^{\mathrm{b}}$ & $3,26^{\mathrm{b}}$ & $3,42^{b}$ & $3,28^{\mathrm{b}}$ & $3,76^{a}$ & 0,001 \\
\hline Requeijão $^{f}$ & $3,45^{\mathrm{a}}$ & $3,26^{\mathrm{b}}$ & $3,28^{\mathrm{b}}$ & $3,35^{\mathrm{b}}$ & $3,26^{\mathrm{b}}$ & $3,80^{\mathrm{a}}$ & 0,001 \\
\hline Molho de tomate ${ }^{f}$ & $3,53^{a, b, c}$ & $3,32^{c}$ & $3,39^{b, c}$ & $3,68^{a, b}$ & $3,37^{b, c}$ & $3,81^{\mathrm{a}}$ & 0,001 \\
\hline Pão de forma ${ }^{\dagger}$ & $3,60^{\mathrm{b}}$ & $3,55^{\mathrm{b}}$ & $3,57^{\mathrm{b}}$ & $3,80^{\mathrm{a}}$ & $3,51^{\mathrm{b}}$ & $3,97^{\mathrm{a}}$ & 0,001 \\
\hline Biscoito água e salf & $3,28^{\mathrm{b}}$ & $3,07^{\mathrm{b}}$ & $3,19^{b}$ & $3,22^{\mathrm{b}}$ & $3,14^{\mathrm{b}}$ & $3,75^{\mathrm{a}}$ & 0,001 \\
\hline Barra de cereal $^{f}$ & $3,36^{\mathrm{b}}$ & $3,32^{\mathrm{b}}$ & $3,26^{\mathrm{b}}$ & $3,27^{\mathrm{b}}$ & $3,26^{\mathrm{b}}$ & $3,84^{\mathrm{a}}$ & 0,001 \\
\hline Cereal matinal ${ }^{f}$ & $3,29^{b}$ & $3,19^{b}$ & $3,09^{\mathrm{b}}$ & $3,17^{\mathrm{b}}$ & $3,18^{b}$ & $3,66^{a}$ & 0,001 \\
\hline Néctar ${ }^{f}$ & $3,25^{\mathrm{a}}$ & $3,10^{\mathrm{b}}$ & $3,03^{b}$ & $3,35^{\mathrm{a}}$ & $3,11^{\mathrm{b}}$ & $3,57^{\mathrm{a}}$ & 0,001 \\
\hline Milho verde ${ }^{f}$ & $3,45^{b, c}$ & $3,39^{b, c}$ & $3,33^{c}$ & $3,72^{a, b}$ & $3,41^{b, c}$ & $3,83^{a}$ & 0,001 \\
\hline Bisnaguinhaf $^{f}$ & $3,36^{\mathrm{a}}$ & $3,37^{a}$ & $3,25^{b}$ & $3,29^{b}$ & $3,30^{\mathrm{b}}$ & $3,66^{a}$ & 0,001 \\
\hline
\end{tabular}

Notas: $\mathrm{p}$-valores da ANOVA. Letras minúsculas iguais na mesma linha indicam que as médias não diferem de acordo com o teste Tukey $(\mathrm{p}<0,05)$.

a Percentual médio de acertos para os nove produtos (0 a 100\%).

b Percentual médio de acertos para cada produto.

" Média da percepção de saudabilidade dos participantes para o conjunto de nove produtos: 1 - "nada saudável a 5 - muito saudável".

d Média da percepção de saudabilidade dos participantes para cada produto individualmente.

" Média da intenção de compra dos participantes para o conjunto de nove produtos: 1 - "eu certamente não compraria a 5 eu certamente compraria".

${ }^{\mathrm{i}}$ Média da intenção de compra dos participantes para cada um dos produtos. 
na presença dos outros quatro modelos de RNF. Na análise de cada alimento individualmente, o percentual médio de acertos na presença do octógono, da lupa, do círculo e do triângulo foi superior ao do GC para os nove alimentos (Tabela 3). Para o semáforo, o percentual médio de acertos foi significativamente superior ao do CG para oito dos nove alimentos.

\section{Percepção de Saudabilidade}

O desempenho dos cinco modelos de RNF foi significativamente superior ao GC, reduzindo as médias de percepção de saudabilidade para o conjunto dos nove alimentos (Tabela 3). Na análise das médias de percepção de saudabilidade para cada alimento isoladamente, a presença do octógono foi a única que reduziu significativamente a percepção de saudabilidade dos participantes para todos os nove alimentos, comparado ao GC. O semáforo apresentou médias inferiores ao GC para quatro alimentos e a lupa, apenas para três alimentos.

\section{Intenção de Compra}

A presença de RNF reduziu a intenção de compra em relação ao GC para o conjunto alimentos investigados (Tabela 3), independente do modelo de RNF. Na análise das médias de intenção de compra para cada alimento individualmente, o octógono e triângulo apresentaram médias significativamente menores do que o GC para os nove alimentos. O círculo apresentou médias inferiores ao GC para oito alimentos. Já a lupa e o semáforo apresentaram médias inferiores ao GC apenas para cinco dos nove alimentos investigados.

\section{Percepção dos Consumidores sobre os Modelos de RNF}

Houve diferença significativa entre os cinco modelos de RNF para os itens "visualizei o selo". O percentual de participantes que declaram ter visualizado a RNF variou de 73,3\% a $83,3 \%$, sendo maior para o semáforo $(83,3 \%)$ e círculo $(79,0 \%)$ do que para o octógono $(73,3 \%)$. A concordância para o item "entendi esse selo" foi maior para o octógono $(4,59)$ quando comparado ao semáforo $(4,41)$.

Os consumidores manifestaram-se favoráveis à presença da RNF, percebendo-a como confiável para aumentar o entendimento das informações nutricionais. Verificou-se alto grau de concordância (médias superiores a 4, em escala de 5 pontos) para todos os itens positivos de percepção dos modelos. Para os itens negativos, como desconforto ou dificuldade para

Tabela 4. Percepção dos participantes em relação a cinco modelos de rotulagem nutricional frontal. Brasil, 2019.

\begin{tabular}{|c|c|c|c|c|c|c|c|}
\hline & $\begin{array}{c}\text { Lupa } \\
n=400\end{array}$ & $\begin{array}{l}\text { Círculo } \\
\mathrm{n}=\mathbf{4 0 0}\end{array}$ & $\begin{array}{c}\text { Octógono } \\
n=400\end{array}$ & $\begin{array}{c}\text { Semáforo } \\
\mathrm{n}=\mathbf{4 0 0}\end{array}$ & $\begin{array}{l}\text { Triângulo } \\
\mathrm{n}=\mathbf{4 0 0}\end{array}$ & $\begin{array}{l}\text { Controle } \\
n=400\end{array}$ & p \\
\hline \multicolumn{8}{|l|}{ Percepção sobre os modelos } \\
\hline Visualizei o selo ${ }^{a}$ & $77,0 \%{ }^{\mathrm{c}, \mathrm{b}}$ & $79,0 \%$ b,a & $73,3 \%^{\mathrm{c}}$ & $83,3 \%{ }^{a}$ & $77,3 \%$ c,b & NA & $0,015^{\mathrm{b}}$ \\
\hline $\begin{array}{l}\text { Encontrei mais rapidamente as informações nutricionais } \\
\text { com esse selo }{ }^{c}\end{array}$ & $4,19^{a}$ & $4,20^{\mathrm{a}}$ & $4,09^{\mathrm{a}}$ & $4,17^{a}$ & $4,18^{\mathrm{a}}$ & NA & $0,710^{\mathrm{d}}$ \\
\hline Entendi esse seloc & $4,49^{a, b}$ & $4,55^{\mathrm{a}, \mathrm{b}}$ & $4,59^{\mathrm{b}}$ & $4,41^{\mathrm{a}}$ & $4,57^{\mathrm{a}, \mathrm{b}}$ & NA & $0,035^{\mathrm{d}}$ \\
\hline $\begin{array}{l}\text { Entendi mais rapidamente as informações nutricionais com } \\
\text { esse selo }{ }^{c}\end{array}$ & $4,35^{\mathrm{a}}$ & $4,37^{\mathrm{a}}$ & $4,31^{\mathrm{a}}$ & $4,35^{\mathrm{a}}$ & $4,37^{\mathrm{a}}$ & NA & $0,919^{d}$ \\
\hline Confiei no seloc & $4,21^{\mathrm{a}}$ & $4,24^{a}$ & $4,09^{a}$ & $4,10^{\mathrm{a}}$ & $4,24^{\mathrm{a}}$ & NA & $0,109^{d}$ \\
\hline $\begin{array}{l}\text { Gostaria de encontrar esse selo nas embalagens de } \\
\text { alimentos }{ }^{c}\end{array}$ & $4,66^{\mathrm{a}}$ & $4,64^{\mathrm{a}}$ & $4,62^{\mathrm{a}}$ & $4,66^{a}$ & $4,70^{\mathrm{a}}$ & NA & $0,706^{d}$ \\
\hline Foi difícil visualizar o selo ${ }^{c}$ & $2,4^{a, b}$ & $2,21^{\mathrm{a}}$ & $2,58^{b}$ & $2,50^{a, b}$ & $2,38^{a, b}$ & NA & $0,009^{d}$ \\
\hline Senti-me desconfortável com esse seloc & $2,42^{\mathrm{a}}$ & $2,38^{\mathrm{a}}$ & $2,48^{\mathrm{a}}$ & $2,35^{\mathrm{a}}$ & $2,34^{\mathrm{a}}$ & NA & $0,724^{\mathrm{d}}$ \\
\hline A presença desse selo me causou medo ${ }^{c}$ & $2,58^{a, b}$ & $2,69^{a, b}$ & $2,66^{a, b}$ & $2,34^{\mathrm{a}}$ & $2,75^{\mathrm{b}}$ & NA & $0,001^{\mathrm{d}}$ \\
\hline
\end{tabular}

NA: não se aplica.

Nota: Letras minúsculas iguais na mesma linha indicam que as médias não diferem de acordo com o teste Tukey $(p<0,05)$.

a Percentual de participantes que respondeu sim para a pergunta.

${ }^{\mathrm{b}} \mathrm{p}$-valores do teste qui-quadrado de Pearson.

"Média de concordância dos participantes em relação a aceitabilidade dos modelos de RNF: "1 - discordo totalmente a 5 - concordo totalmente".

dp-valores da ANOVA. 
identificação do modelo, observou-se baixa concordância (médias inferiores a 2,5). Apesar da baixa concordância, a média do octógono $(2,58)$ para o item "foi difícil visualizar o selo" foi superior à média do círculo $(2,21)$. Para o item "a presença desse selo me causou medo", a média do semáforo $(2,34)$ foi inferior à observada para o triângulo $(2,75)$.

\section{DISCUSSÃO}

O entendimento do conteúdo nutricional é considerado crucial para avaliar a efetividade da rotulagem nutricional ${ }^{4,5}$. Os modelos de RNF de advertências (octógono, triângulo, círculo) apresentaram desempenho superior ao semáforo no que se refere ao entendimento do conteúdo nutricional. A superioridade das advertências em relação ao semáforo já havia sido relatada em estudos anteriores ${ }^{6,10,21,27}$.

Diferente do que havia sido reportado por estudo anterior ${ }^{9}$, não se observou melhor desempenho do modelo da lupa em relação ao semáforo para este quesito e vários fatores podem explicar esses resultados. O processamento da informação, a familiaridade com o símbolo utilizado, a capacidade do modelo em capturar a atenção do consumidor e a sua cor são fatores já evidenciados como importantes influenciadores do entendimento do conteúdo nutricional ${ }^{9,27,28}$.

Em relação ao processamento da informação, o semáforo não apresenta a mesma objetividade das advertências, que informam apenas os nutrientes presentes em alto teor no alimento. Desta forma, um alimento pode ter, por exemplo, um campo vermelho (alto) e dois verdes (baixo), o que pode aumentar a percepção de saudabilidade do mesmo, sendo essa uma possível limitação do modelo ${ }^{6,10}$.

Já em relação à familiaridade, a lupa é o único modelo que não é amplamente utilizado ou padronizado, sendo menos familiar ao consumidor do que as advertências ${ }^{9}$. A familiaridade ao símbolo utilizado na RNF é essencial para se estabelecer uma comunicação rápida e clara, possibilitando melhor entendimento. De acordo com o modelo de processamento da informação humana, os sinais de alertas familiares e padronizados internacionalmente são o triângulo (sinal mais associado ao risco), o octógono (associado a placa de trânsito pare), o semáforo e o círculo vermelho (utilizados no trânsito) ${ }^{28}$.

O entendimento do conteúdo nutricional também está relacionado à captura de atenção, mensurada pelo tempo necessário para o consumidor localizar e visualizar a RNF e pelo tempo requerido para identificar os nutrientes presentes em excesso ${ }^{27}$. O semáforo e a lupa são modelos de RNF que exigem maior tempo de captura de atenção em comparação com as advertências ${ }^{9}$. Os modelos de advertência apresentam imagens que se repetem a cada nutriente presente em excesso, chamando mais atenção do consumidor quando comparados aos modelos com imagem única ${ }^{29}$.

A captura de atenção é também influenciada pela cor, apresentação em imagem ou texto, posição e símbolo utilizado na $\mathrm{RNF}^{27-29}$. O melhor desempenho das advertências (octógono, triângulo e círculo) em relação ao semáforo, para o entendimento, pode estar relacionado com a cor, já que o preto apresenta captura de atenção mais rápida, seguida do vermelho ${ }^{27}$.

Todos os modelos de RNF reduziram a percepção de saudabilidade dos alimentos e a intenção de compra se comparados ao GC, entretanto, na análise individual, o semáforo e a lupa reduziram a percepção de saudabilidade e intenção de compra de um número menor de alimentos em comparação às advertências. Ao perceber um alimento como não saudável, espera-se que o consumidor reduza a intenção de compra ${ }^{8}$. Uma das explicações para o desempenho inferior do semáforo nestes dois quesitos, pode ser a presença da informação "baixo conteúdo" na cor verde, cor normalmente associada a referências positivas, o que pode aumentar percepção de saudabilidade do alimento mesmo com a presença de alto teor de outro nutriente crítico e consequentemente aumentar a intenção de compra ${ }^{6,9,10}$. Já o desempenho inferior da lupa pode estar relacionado ao fato deste ser o único modelo 
que não tem design familiar ao consumidor, exigindo mais esforço de interpretação para o julgamento quanto à saudabilidade do alimento e a decisão de compra, além de não ser um modelo associado ao risco, como as advertências, as quais já se mostraram capazes de reduzir a intenção de compra em estudos anteriores ${ }^{9,17,21,26}$.

A percepção dos participantes foi favorável à presença da RNF nas embalagens dos alimentos, entendendo a RNF como confiável e de fácil visualização e interpretação para melhorar o entendimento do conteúdo nutricional dos alimentos, conforme já observado em estudos semelhantes ${ }^{9,10,30}$.

O percentual de participantes que declarou ter visualizado o semáforo e o círculo foi superior ao observado para o octógono. A média de concordância para o item "foi difícil visualizar o selo" também foi maior para o octógono em comparação ao círculo. Estes achados subjetivos, mensurados a partir da percepção dos participantes, diferem de estudo anterior, no qual a dificuldade de visualização foi mensurada de forma objetiva, por meio do tempo necessário para visualizar a RNF com a utilização de software, quando o círculo exigiu mais tempo em comparação com o semáforo e o octógono ${ }^{9}$.É esperado que o modelo de mais fácil visualização também apresente melhor desempenho quanto ao entendimento do conteúdo nutricional, entretanto, o octógono foi o modelo de advertência que apresentou maior percentual de acertos $(62,4 \%)$ para o entendimento do conteúdo nutricional. A contradição entre resultados obtidos por medidas objetivas e subjetivas foi reportada por estudo anterior, sugerindo que a percepção dos consumidores pode não refletir com precisão o desempenho dos modelos de RNF9 .

Em relação ao item "a presença desse selo me causou medo" é preciso cautela na interpretação dos resultados. Estudos sugerem limitações da abordagem das emoções básicas em detectar diferentes aspectos da experiência emocional ${ }^{26}$. A maior média deste quesito para o triângulo comparado ao semáforo pode estar, por exemplo, alinhada aos estudos da psicologia que reportam que o medo é uma emoção ativada por situações potencialmente ameaçadoras ou por perigos reais ${ }^{31}$. Além disso, também pode estar alinhada ao modelo de comunicação humana, que reporta ser o triângulo o sinal mais associado à $\operatorname{risco}^{27,28}$. As advertências (octógono, círculo e triângulo) não diferiram entre si neste quesito, corroborando com o modelo da comunicação humana que reporta serem sinais de alerta mais familiares aos consumidores, sendo também mais comumente associados ao risco ${ }^{26}$. Sugere-se que outros estudos aprofundem o estudo das emoções associadas à presença a RNF, não se restringindo ao medo, avaliando com detalhes seus fatores e os comportamentos gerados ${ }^{31}$.

No que se refere às limitações deste estudo, sugere-se que pesquisas futuras incluam a simulação de fatores presentes na situação real de compra, tais como tempo limitado, presença de alegações nutricionais e publicidade no rótulo dos alimentos, além de número maior de alimentos, incluindo produtos saudáveis e não saudáveis.

O presente estudo foi realizado com uma amostra robusta e diversa em termos de sexo, faixa etária, escolaridade, renda e região do país. O controle das dessas variáveis garantiu homogeneidade entre os grupos, as quais não apresentaram diferença estatística. Por fim, cabe salientar que a importância de fatores relacionados à escolha alimentar como, por exemplo o preço, a facilidade de preparo, a proximidade do local de compra, a preferência por alimentos mais saudáveis, não diferiu entre os grupos de exposição e controle, não sendo, portanto, fatores influenciadores do desempenho dos modelos de RNF na amostra estudada.

\section{Conclusão}

Este estudo evidenciou que a RNF aumenta o entendimento do conteúdo nutricional, reduz a percepção de saudabilidade e a intenção de compra de alimentos com alto conteúdo de açúcares, gorduras saturadas e sódio. Os modelos de RNF de advertências (octógono, triângulo e círculo) apresentaram desempenho superior ao semáforo para o entendimento. 
O modelo da lupa apresentou resultados menos consistentes do que os modelos de advertência (octógono, triângulo e círculo). Em relação à percepção, os resultados revelaram que os consumidores são favoráveis à presença da RNF nos alimentos.

Os resultados deste estudo trazem importantes subsídios aos formuladores de políticas púbicas, reforçando a necessidade e vantagens da adoção da RNF no Brasil. Tal medida é urgente em um cenário onde estudos já apontam aumento de preços dos alimentos saudáveis e barateamento de produtos ultraprocessados nos próximos anos ${ }^{32}$. A escolha do modelo de RNF a ser adotado no país deve ser criteriosa e considerar as evidências científicas disponíveis, buscando escolher o modelo com maior potencial de bom desempenho, alinhado às particularidades da população que dele se beneficiará.

\section{REFERÊNCIAS}

1. WHO. Report of the commission on ending childhood obesity. Geneva: World Health Organization; 2016. 68p.

2. WHO. Technical Meeting on Nutrition Labelling for Promoting Healthy Diets. Lisbon: World Health Organization; 2015.

3. Gomes ASL. Letramento Científico: um indicador para o Brasil. São Paulo: Instituto Abramundo; 2015. $94 \mathrm{p}$

4. Grunert KG, Wills JM. A review of European research on consumer response to nutrition information on food labels. J Public Health. 2007;15(5):385-99. https://doi.org/10.1007/s10389-007-0101-9

5. Grunert KG, Wills JM, Fernández-Celemín L. Nutrition knowledge, and use and understanding of nutrition information on food labels among consumers in the UK. Appetite. 2010;55(2):177-89. https://doi.org/10.1016/j.appet.2010.05.045

6. Arrúa A, Machín L, Curutchet MR, Martínez J, Antúnez L, Alcaire F et al. Warnings as a directive front-of-pack nutrition labelling scheme: comparison with the Guideline Daily Amount and traffic-light systems. Public Health Nutr. 2017;20(13):2308-17. https://doi.org/10.1017/S1368980017000866

7. Ares G, Varela F, Machin L, Antúnez L, Giménez A, Curutchet MR et al. Comparative performance of three interpretative front-of-pack nutrition labelling schemes: Insights for policy making. Food Qual Prefer. 2018;68:215-25. https://doi.org/10.1016/j.foodqual.2018.03.007

8. Ares G, Aschemann-Witzel J, Curutchet MR, Antúnez L, Machín L, Vidal L et al. Nutritional warnings and product substitution or abandonment: Policy implications derived from a repeated purchase simulation. Food Qual Prefer. 2018;65:40-8. http://doi.org/10.1016/j. foodqual.2017.12.001

9. Deliza R, Alcantara M, Pereira R, Ares G. How do different warning signs compare with the guideline daily amount and traffic-light system? Food Qual Prefer. 2020;80:1168-70. https://doi.org/10.1016/j.foodqual.2019.103821

10. Khandpur N, Sato PM, Mais LA, Martins APB, Spinillo CG, Garcia MT et al. Are front-of-package warning labels more effective at communicating nutrition information than traffic-light labels? A randomized controlled experiment in a Brazilian sample. Nutrients. 2018;10(6):688. https://doi.org/10.3390/nu10060688

11. Chile. Ley núm. 20.606. Sobre composición nutricional de los alimentos y su publicidad. Ministerio de Salud. 6 jun. 2012. Disponível em: https://www.leychile.cl/Navegar?idNorma=1041570

12. México. PROYECTO de Modificación a la Norma Oficial Mexicana NOM-051-SCFI/SSA1-2010: especificaciones generales de etiquetado para alimentos y bebidas no alcohólicas preenvasados-Información comercial y sanitaria, publicada el 5 de abril de 2010. Diário Oficial de la Federación. 11 out. 2019. Disponível em: https://dof.gob.mx/nota_detalle.php?codigo=5575205\&fecha=11/10/2019

13. Perú. Ley de promoción de la alimentación saludable para niños, niñas y adolescentes, y su Reglamento aprobado por Decreto Supremo No 017-2017-SA. Diário Oficial El Peruano. 2017. Disponível em: https://busquedas.elperuano.pe/normaslegales/decreto-supremo-queaprueba-el-reglamento-de-la-ley-n-30021-decreto-supremo-n-017-2017-sa-1534348-4/

14. Uruguay. Decreto interno no 001-3/13061/2017/VF. Ministério de la Salud Pública. 29 ago. 2018. Disponível em: http://actbr.org.br/uploads/arquivos/Decreto-Presidencial-Rotulagem-uruguai.pdf 
15. Ministério da Saúde. Agência Nacional de Vigilância Sanitária. Resolução de Diretoria Colegiada $\mathrm{n}^{\circ} 429$, de 8 de outubro de 2020. Dispõe sobre a rotulagem nutricional dos alimentos embalados. Diário Oficial da União. 9 out 2020; Seção 1:106.

16. Health Canada. Consumer research on front of package nutrition labeling. Montréal: Léger; 2018. Diponível em: http://epe.lac-bac.gc.ca/100/200/301/pwgsc-tpsgc/por-ef/health/2018/073-17-e/report.pdf

17. Goodman S, Vanderlee L, Acton R, Mahamad S, Hammond D. The impact of front-of-package label design on consumer understanding of nutrient amounts. Nutrients. 2018;10(11):1624. https://doi.org/10.3390/nu10111624

18. Heitor SFD, Estima CCP, Neves FJ, Aguiar AS, Castro SS, Ferreira JES. Tradução e adaptação cultural do questionário sobre motivo das escolhas alimentares (Food Choice Questionnaire - FCQ) para a língua portuguesa. Ciênc Saúde Coletiva. 2015;20(8):2339-46. http://doi.org/10.1590/1413-81232015208.15842014

19. Mejean C, Macouillard P, Péneau S, Hercberg S, Castetbon K. Consumer acceptability and understanding of front-of-pack nutrition labels. J Hum Nutr Diet. 2013;26(5):494-503. https://doi.org/10.1111/jhn.12039

20. Ducrot P, Méjean C, Julia C, Kesse-Guyot E, Touvier M, Fezeu L et al. Effectiveness of front-of-pack nutrition labels in french adults: results from the NutriNet-Santé cohort study. PLoS One. 2015;10(10):e0140898. https://doi.org/10.1371/journal.pone.0140898

21. Taillie LS, Hall MG, Popkin BM, Ng SW, Murukutla N. Experimental Studies of Front-of-Package Nutrient Warning Labels on Sugar- weetened Beverages and Ultra-Processed Foods: A Scoping Review. Nutrients. 2020;12(2):569. https://doi.org/10.3390/nu12020569

22. Organisation for Economic Co-operation and Development. The heavy burden of obesity: the economics of prevention. Paris: OECD Health Policy Studies; 2019. https://doi.org/10.1787/67450d67-en

23. Agência Nacional de Vigilância Sanitária. Relatório preliminar de análise de impacto regulatório sobre rotulagem nutricional. Brasília, DF: Anvisa; 2018. 249 p.

24. Instituto Brasileiro de Geografia e Estatística. Pesquisa de orçamentos familiares 2008-2009: análise do consumo alimentar pessoal no Brasil. Rio de Janeiro: IBGE; 2011. 150 p.

25. Nielsen J. Usability engineering. Burlington: Morgan Kaufmann; 1993. 362 p.

26. Michelini Y, Acuña I, Guzmán JI, Godoy JC. LATEMO-E: A Film Database to Elicit Discrete Emotions and Evaluate Emotional Dimensions in Latin-Americans. Trends Psychol. 2019;27(2):473-90. http://doi.org/10.9788/tp2019.2-13

27. Cabrera M, Machín L, Arrúa A, Antúnez L, Curutchet MR, Giménez A et al. Nutrition warnings as front-of-pack labels: Influence of design features on healthfulness perception and attentional capture. Public Health Nutr. 2017;20(18):3360-71. https://doi.org/10.1017/s136898001700249x

28. Wogalter MS, Dejoy D, Laughery KR. Warnings and risk communication. Boca Raton: CRC Press; 1999.357 p.

29. Pieters R, Wedel M. Attention capture and transfer in advertising: brand, pictorial, and text-size effects. J Mark. 2004;68(2):36-50. https://doi.org/10.1509\%2Fjmkg.68.2.36.27794

30. Julia C, Péneau S, Buscail C, Gonzalez R, Touvier M, Hercberg S et al. Perception of different formats of front-of-pack nutrition labels according to sociodemographic, lifestyle and dietary factors in a French population: Cross-sectional study among the NutriNet-Santé cohort participants. BMJ Open. 2017;7(6):e016108. https://doi.org/10.1136/bmjopen-2017-016108

31. Baptista A, Carvalho M, Lory F. O medo, a ansiedade e as suas perturbações. Psicologia. 2005;19(1-2):267-77.

32. Maia EG, Passos CM, Levy RB, Martins APB, Mais LA, Claro RM. What to expect from the price of healthy and unhealthy foods over time? The case from Brazil. Public Health Nutr. 2020;23(4):579-88. https://doi.org/10.1017/S1368980019003586

Financiamento: Estudo financiado pelo Conselho Nacional de Desenvolvimento Científico e Tecnológico (CNPq - Processo 441760/2017-1)

Contribuição dos Autores: Concepção e planejamento do estudo: LMB, JP, NT, MBG. Análise e interpretação dos dados: LMB, JP, NT, MBG. Redação do manuscrito: LMB. Revisão crítica do conteúdo: LMB, JP, NT, MBG. Todas as autoras participaram da aprovação da versão final do manuscrito e assumem responsabilidade pública pelo conteúdo do artigo.

Conflito de Interesses: Os autores declaram não haver conflito de interesses. 